\title{
Comparative Study of Testing Tools Blazemeter and Apache Jmeter
}

\begin{abstract}
Pirah Memon ${ }^{1}$, Tahseen Hafiz ${ }^{2}$, Sania Bhatti ${ }^{2}$, Saman Shahid Qureshi ${ }^{1}$
Abstract:

Automated Testing plays a vital role in the entire development of software. Due to growing requirements of an automated testing diverse range of testing tools are available. From litera-ture, it is observed that a number of automated testing tools are studied and compared. How-ever, this is the first time that Apache JMeter and BlazeMeter are compared. The objective of this paper is to compare load testing tools: Apache JMeter and BlazeMeter based on the criteria such as performance, latency, size, error percent, duration count, number of hits and re-sponse time. This paper focuses on the analysis of the performance and functionality that minimizes the software cost and resources. After performing experiments, it is proved that the performance of BlazeMeter is better than Apache JMeter with respect to all parameters.
\end{abstract}

Keywords: BlazeMeter; Apache JMeter; Automated software testing.

\section{Introduction}

Software testing is used to find out the errors in the software product. Software testing identifies the product completeness, correctness and also used to improve the product quality. Testing does not guarantee the error-free software. Rather, testing helps to debug the error within the software. There are various approaches for testing, which depend upon the software requirements, category of software and available resources .In simple word, testing is to "verify the product and evaluate it". Where the term verifies is something that the tester wants to match the requirements with the actual product and the response of product with the behavior in action to the analysis of the tester. Although most of the intellectual properties are identical to the inspections of the requirements the word testing is concerned with dynamic analysis of the product. Testing helps us improve the quality of the software. The quality of the software can be improved by involving the non-functional attributes according to the standard of ISO-9126. Testing is about verifying and validating software if it is working as it is intended to design. It involves testing of a product using static and dynamic methodologies because of human mistakes, manual designs. Hence the quality of the software can be achieved by performing the quality assurance activities. It is usual for the developer to spend $40 \%$ of the software cost on testing. For example bank transaction monitor, control can cost 3 to 5 times as much as all other activities are combined due to the antagonistic nature of testing the developer does not consider the notations in its development of software.

\section{Related Work}

There has been a significant work on the comparative analysis of HP LoadRunner and Apache JMeter in literature but limited work on BlazeMeter. The limitation of the paper proposed by V.Chandel [1] is that they only described the Apache JMeter HP LoadRunner but does not depict the real time results. The

\footnotetext{
${ }^{1}$ Institute of information and communication Technology MehranUniversity of Engineering and Technology Jamshoro, Pakistan

${ }^{2}$ Department of Software Engineering, Mehran University of Engineering and Technology, Jamshoro, Pakistan Corresponding Email: pirahmemon01@gmail.com
} SJCMS | P-ISSN: 2520-0755 | E-ISSN: 2522-3003 ( 2018 Sukkur IBA University - All Rights Reserved 
automated web services testing tools presented in study [2] are very interesing as it describes the Apache JMeter ,SoapUI and Storm in detail but they do not justify that among them which tool is better. In paper [3] M.S Sharmila. et al. Discussed the Apache JMeter in a well mannered way but the factors for comparison with other tools are not focused. In study [4] K. Tirghoda illustrated the Apache tool in detail but any script for web services which are being tested using the Apache tool is not generated. Sadiq et al. [5] uses response time ,throughput, latency, scalability and resource utilization for Apache JMeter but does not delineate the security issues related to Apache JMeter. B. Patel, et al. [6] compared two performance testing tools i.e LoadRunner and JMeter. They compared the parameters such as load generating capacity, installation, download proficiency result reporting, cost, technicality of software and reliability. The comparative analysis is done between HP LoadRunner and Apache JMeter by R. B. khan [7] but author only targets the websites LOAN calculator and BMI calculator because of not enough traffic on these websites. Authors in [8] put light on two load testing tools: Sikuli and Commercial Tool for acceptance testing. They compared on static properties and industrial traffic management system but there is no statistical difference between these tools; both performed the same automated testing. In study [9] the empirical analysis of web service testing tools is performed with the technical features and the comparison is completed on the basis of performance only. In a recent study [10] the comparative analysis is done among the Selenium, Soap UI, HP QTP/UFT and Test Complete on the basis of different features. Authors use the 3-point scale, i.e. good, average and bad in comparison. The results are presented in the form of graphs based on the calculated values for selected tools and soap is considered as the best tool among them.

\section{Apache JMeter}

Apache JMeter [11] is an Apache open source software, a pure Java application, designed to perform the functional behavior and performance testing specially on web applications that further expand into the other applications on both static and dynamic resources (web services SAOP/RESET) web dynamic languages PHP, Java and Asp.net files but JMeter does not execute the Java script found within the html pages nor it does render the html pages as browser does. It can also be used to graphically analyze the performance or to test the serverlscriptlobject behavior under heavy concurrent load.

Figure 1 shows that as the request is sent by the user is directly acknowledged by the server, the server responses to the user's request, then JMeter collects the data and manipulates the statistical information of further task will be completed and the results will be displayed.

Figure 2 defines the test plan for starting testing of facebook.

Test plan requires four elements http default request, http, cookie manager, a listener and graph result.

Figure 3 describes the number of users along with the test start and end time with a loop count at each thread while the users are generated.

In figure 4, the black labels show the data of the facebook, the blue line defines the slight change in average number of users, pink line defines the no change occur in the median while testing. However, red line shows that deviation changing occur constantly, whereas the green line output increases as the number of users increases. On the y-axis the maximun time is 63068 milliseconds is the time required for completing the facebook uniform resource locator test.Here the graph result depicts the visual model of facebook samples that can be read and written from a file. 

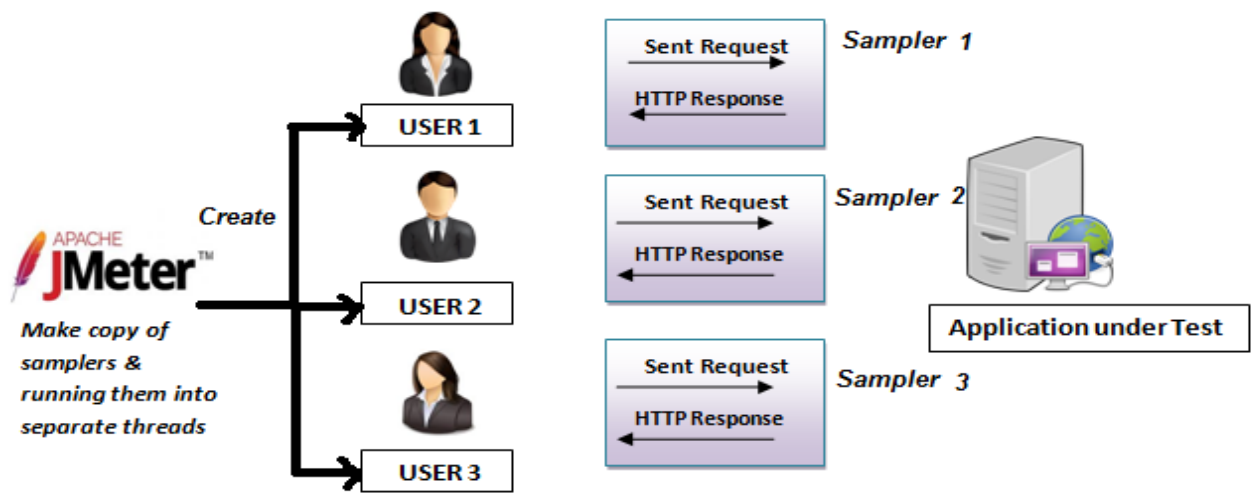

Fig. 1. Working mechanism of Apache JMeter [3].

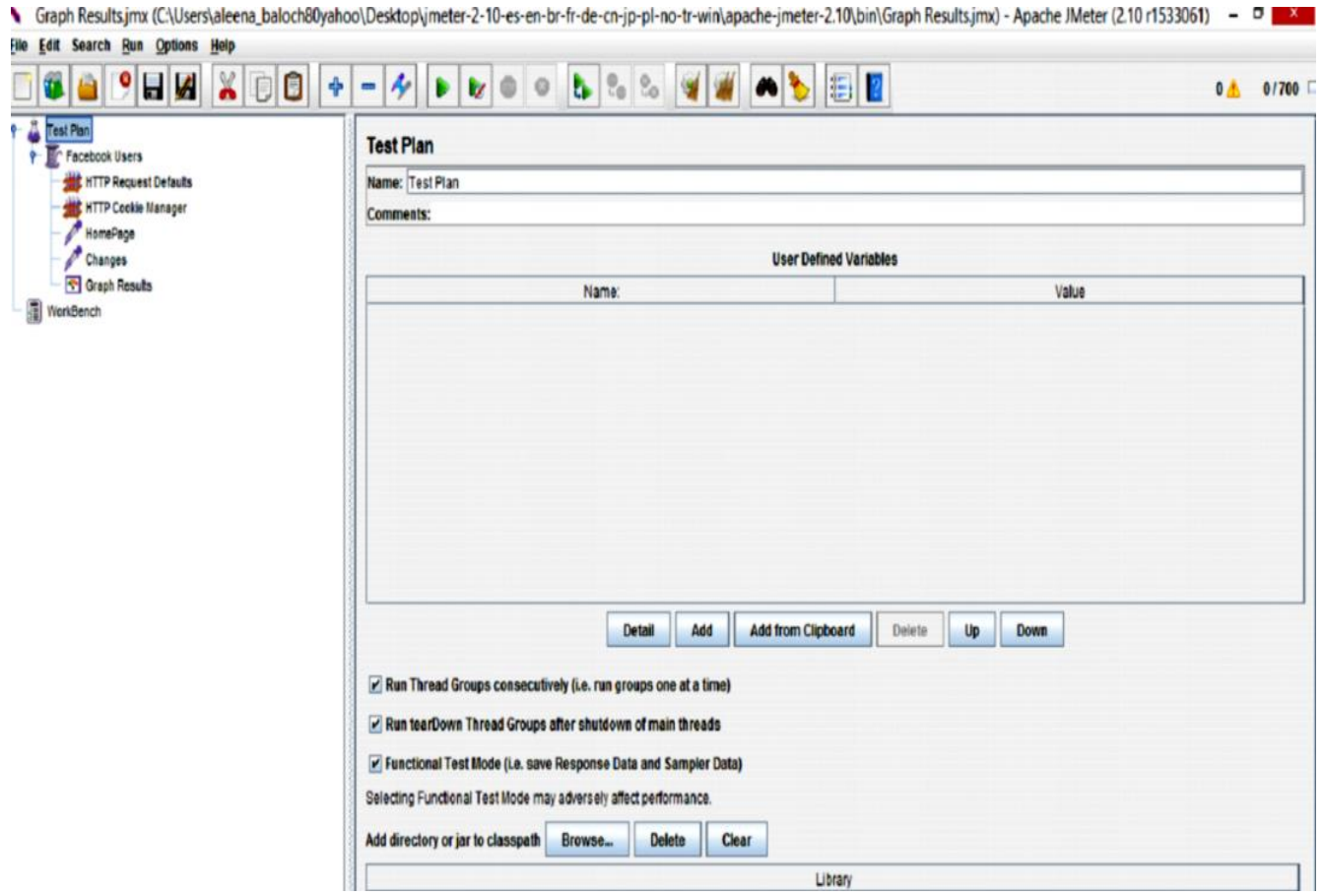

Fig. 2. Facebook url test with Apache JMeter. 


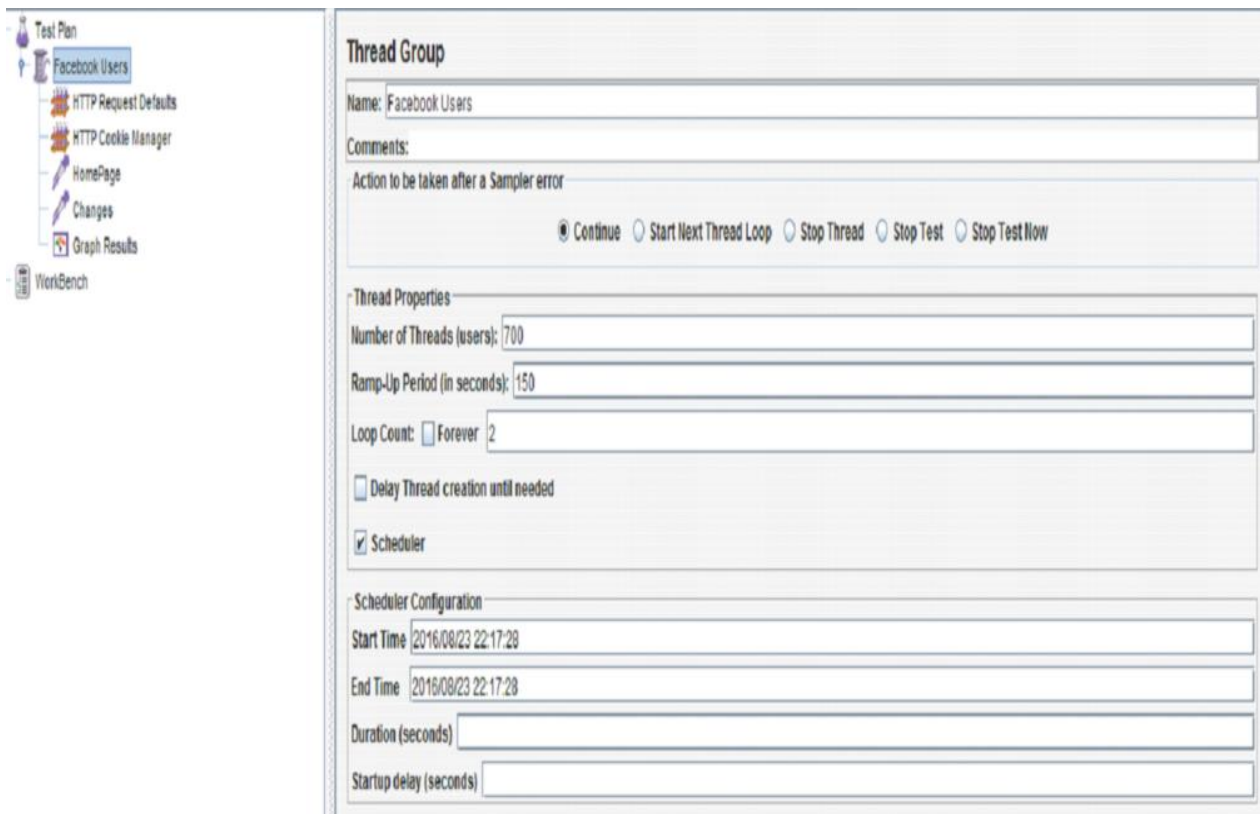

Fig. 3. Thread group of facebook url test with Apache JMeter.

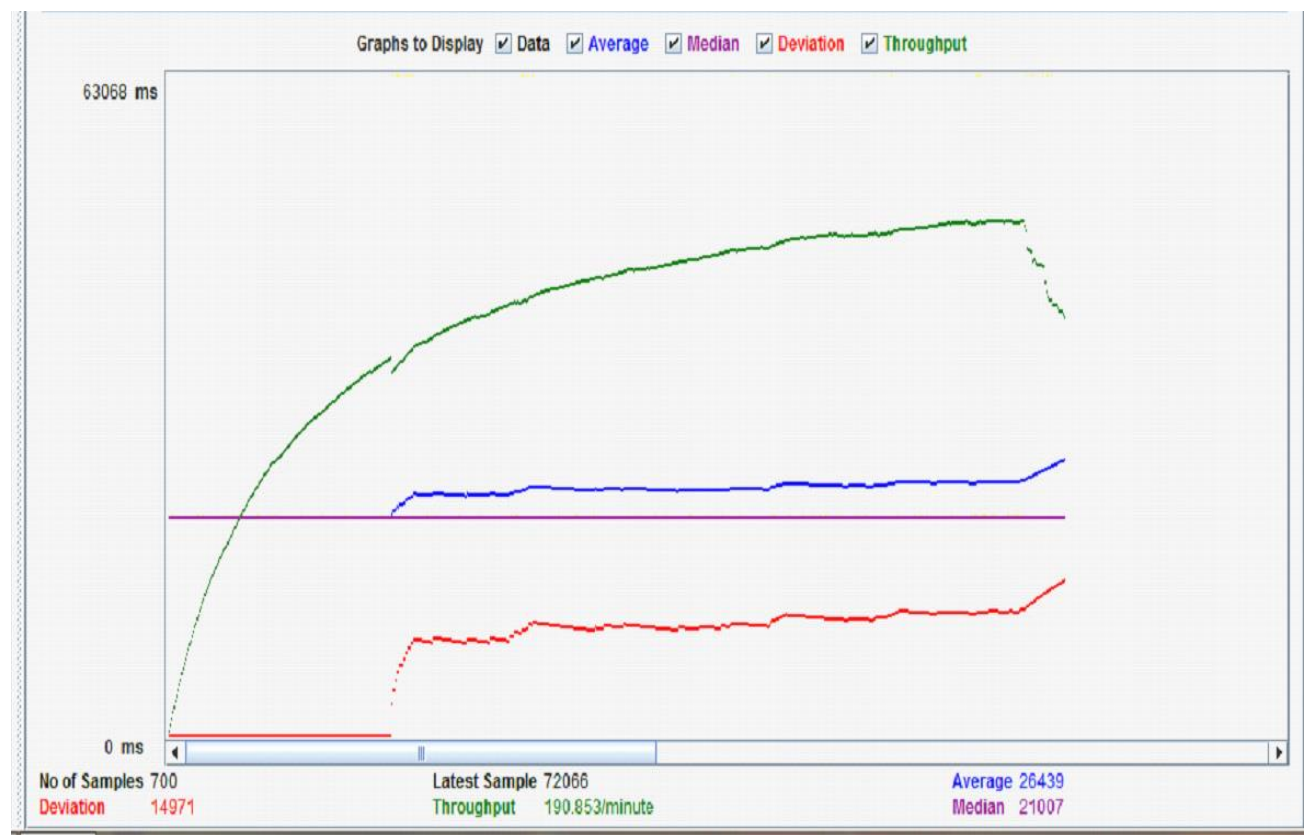

Fig. 4. Graphical depiction of facebook url test. 


\section{Blazemeter Testing Tool}

BLAZEMETER [12] is an enterprise tool fully compatible with Apache JMeter. It provides the developer's simple integrated tool into their native development environment. Blaze Meter is used to test the mobile, web applications; web site, web services, and database testing that can simulate thousands of users. The objective of this paper is to conduct a comparative analysis of social website, facebook to improve the performance of the social websites by making the system more reliable at a less response time.

\section{Results And Discussion}

The goal of this work is to improve the performance of social website "facebook" by performing load testing with BlazeMeter instead of Apache JMeter. Because Apache JMeter fails when the scalability of the product is increased and the behavior is inflexible that means modifications cannot be applied after performing testing even if they are needed. The performance of the testing tools is judged on the basis of the following factors.

Flexibility: It is the non- functional quality attributes of software engineering. Flexibility refers to how easily the changes can be accommodated in the system.

Scalability: Scalability refers to the how easily the system can be expanded by increasing the number of users.

Performance: Performance is the ability of the system to perform the task.

Load Controller: The device used to regulate the amount of power that a load can consume. It can be used by third party energy or utility to reduce the customer energy demands at the certain time.

Reliability: The ability of the system to perform failure free operation for a specified time in a specified environment.
Aggregate Reports: It allows reviewing an overview of administrative information for various settings and status.

Latency Time: It is defined as the amount of time a message takes to reverse a system or to reach a designation.

Table 1. Comparison of testing tools based on various factors.

\begin{tabular}{|l|l|c|c|}
\hline & \multicolumn{1}{|c|}{ Factors } & BlazeMeter & $\begin{array}{c}\text { Apache } \\
\text { JMeter }\end{array}$ \\
\hline 1 & Flexibility & Yes & Yes \\
\hline 2 & Scalability & Yes & Yes \\
\hline 3 & Performance & Yes & Yes \\
\hline 4 & Load controller & Yes & No \\
\hline 5 & Reliability & Yes & Yes \\
\hline 6 & $\begin{array}{l}\text { Aggregate } \\
\text { reports }\end{array}$ & Yes & No \\
\hline 7 & Latency time & No & Yes \\
\hline
\end{tabular}

Table 02, as described in Section IV, defines that at each point the virtual number of users is generated with minimum response time at an average bandwidth of $20 \mathrm{~KB}$ with zero percent error and also shows that the test pass successfully.

TABLE 2. Result of facebook url test with $0 \%$ error and 50 virtual users.

\begin{tabular}{|l|l|l|l|l|l|}
\hline $\begin{array}{l}\text { Max. } \\
\text { Users }\end{array}$ & $\begin{array}{l}\text { Avg. } \\
\text { Thro } \\
\text { ughp } \\
\text { ut }\end{array}$ & $\begin{array}{l}\text { Er } \\
\text { ro } \\
\text { r }\end{array}$ & $\begin{array}{l}\text { Avg.R } \\
\text { espons } \\
\text { e Time }\end{array}$ & $\begin{array}{l}\text { 90\%R } \\
\text { espons } \\
\text { e Time }\end{array}$ & $\begin{array}{l}\text { Avg. } \\
\text { Band } \\
\text { widt } \\
\text { h }\end{array}$ \\
\hline $50 \mathrm{VU}$ & $\begin{array}{l}0.78 \\
\text { Hits/s }\end{array}$ & $\begin{array}{l}0 \\
\%\end{array}$ & $\begin{array}{l}174.5 \\
\text { (MS) }\end{array}$ & $\begin{array}{l}211 \\
\text { (MS) }\end{array}$ & $\begin{array}{l}20.15 \\
\text { KB }\end{array}$ \\
\hline
\end{tabular}

Figure 5 shows the $0 \%$ error means the test pass successfully with fifty users and maximum number of hits which are 0.86 per milliseconds. 


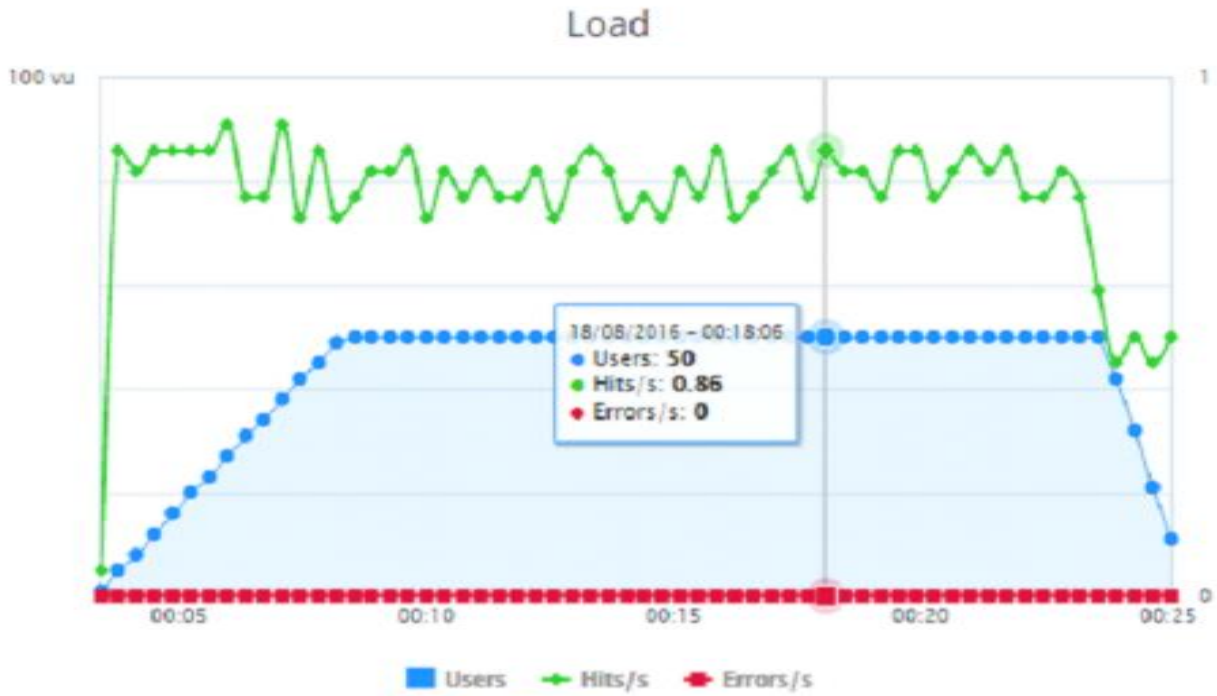

Fig. 5. Result of facebook url test with BlazeMeter for number of hits.avg.

TABLE 3. Samples of Facebook URL test with response time.

\begin{tabular}{|l|l|l|l|l|l|l|l|}
\hline $\begin{array}{l}\text { Element } \\
\text { Label }\end{array}$ & Samples & $\begin{array}{l}\text { Avg. } \\
\text { Response } \\
\text { Time } \\
\text { (Ms) }\end{array}$ & $\begin{array}{l}\text { Avg. } \\
\text { Hits/s }\end{array}$ & $\begin{array}{l}\text { 99\%Line } \\
\text { (Ms) }\end{array}$ & $\begin{array}{l}\text { Min. } \\
\text { Response } \\
\text { Time } \\
(\mathbf{m s})\end{array}$ & $\begin{array}{l}\text { Avg. } \\
\text { Bandwidth } \\
\text { Bytes/s }\end{array}$ & $\begin{array}{l}\text { Error } \\
\text { Rate }\end{array}$ \\
\hline Facebook & 19612 & 187.3 & 16.34 & 624 & 155 & 2931 & $0 \%$ \\
\hline
\end{tabular}

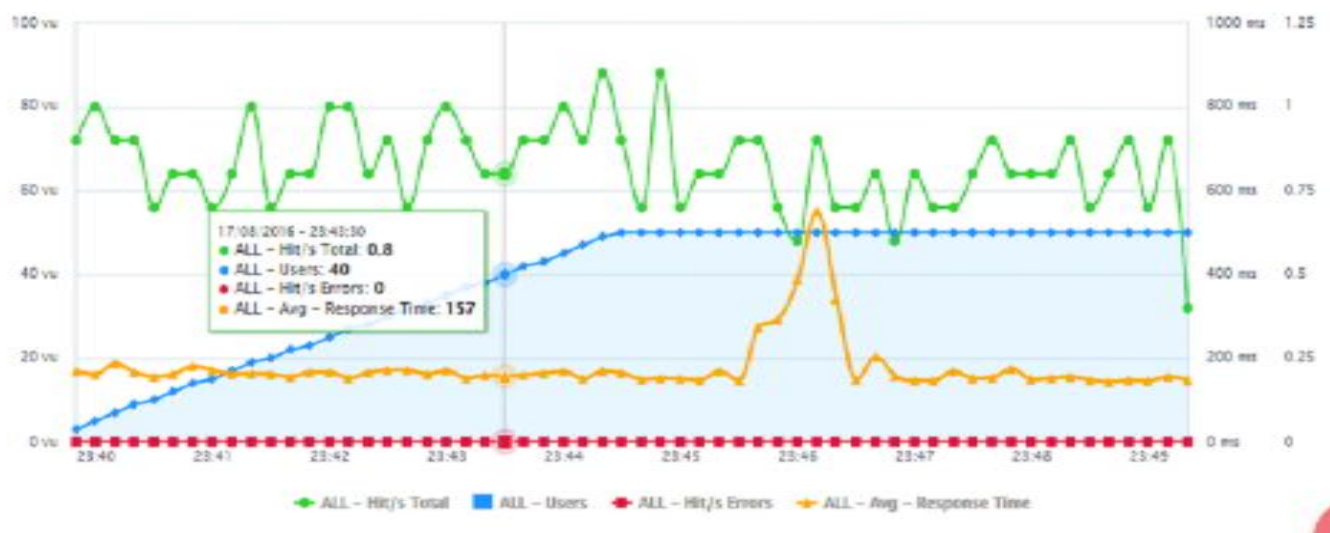

Fig. 6. Result of facebook BlazeMeter for latency url test with BlazeMeter for latency.

\footnotetext{
Sukkur IBA Journal of Computing and Mathematical Sciences - SJCMS | Volume 2 No. 1 January - June 2018 @ Sukkur IBA University
} 
Figure 6 depicts that at each point the test is carried by the virtual users with maximum throughput and average response time of 157 milliseconds.

In Table 3 column 2 describes the number of samples with value 19612, column 3 shows the average response time to value of 187.3 milliseconds, column 4 depicts the average number of hits, column 5 defines the time, in milliseconds, column 6 illustrates the minimum response time, column 7 the average bandwidth and column 8 represents the error $0 \%$ means test pass successfully.

\section{Conclusion}

Currently, software testing has become the necessity for the organizations. Because it saves both time and money. Apache JMeter and BlazeMeter are very efficient for encountering the performance testing of software. From experiments it is appearant that BlazeMeter tool is more efficient as compared to Apache JMeter. It has a simple, clean User interface that shows what's going on without confusion And too much effort and it offers straightforwardness with its Uniqueness. Moreover, it is free of cost and possesses effective Portability with 100\% Java purity. Both of them are open Source projects and have merits, but neither is ideal. Because the experiments are performed with a limited number of users and more experiments are required to be performed with increased number of users.

\section{REFERENCES}

[1] V. Chandel et al., "Comparative Study of Testing Tools: Apache JMeter and Load Runner," International Journal of Computing and Corporate Research, 2013.

[2] G. Murawski, et al., "Evaluation of load testing tools," 2014.
[3] M. S. Sharmila and E. Ramadevi, "Analysis of performance testing of web application," International Journal of Advanced Research in Computer and Communication Engineering, 2014.

[4] K. Tirghoda, "Web Services Performance Testing Using Open Source Apache Jmeter," International Journal of Scientific \& Engineering Research, vol. 3, 2012.

[5] M. Sadiq, et al., "A Survey of Most Common Referred Automated Performance Testing Tools," ARPN Journal of Science and Technology, vol. 5, pp. 525-536, 2015.

[6] B. Patel, et al., "A Review Paper on Comparison of SQL Performance Analyzer Tools: Apache JMeter and HP LoadRunner," 2014.

[7] R. B. Khan, "Comparative Study of Performance Testing Tools: Apache JMeter and HP LoadRunner," Ed. 2016.

[8] E. Borjesson and R. Feldt, "Automated system testing using visual GUI testing tools: A comparative study in the industry," in 2012 IEEE Fifth International Conference on Software Testing, Verification and Validation, 2012, pp. 350-359.

[9] S. Sharma and A. K. Sharma, "Empirical Analysis of Web Service Testing Tools".

[10] M. Imran, et al., "A Comparative Study of QTP and Load Runner, Automated Testing Tools and their Contributions to Software Project Scenario," 2016.

[11]A. S. Foundation. 2017-1-19. Apache JMeter. Available: http://jmeter.apache.org/.

[12] A. Girmonsky. (2016, 01-12-2016). BlazeMeter. Available: http://blazemeter.com/. 